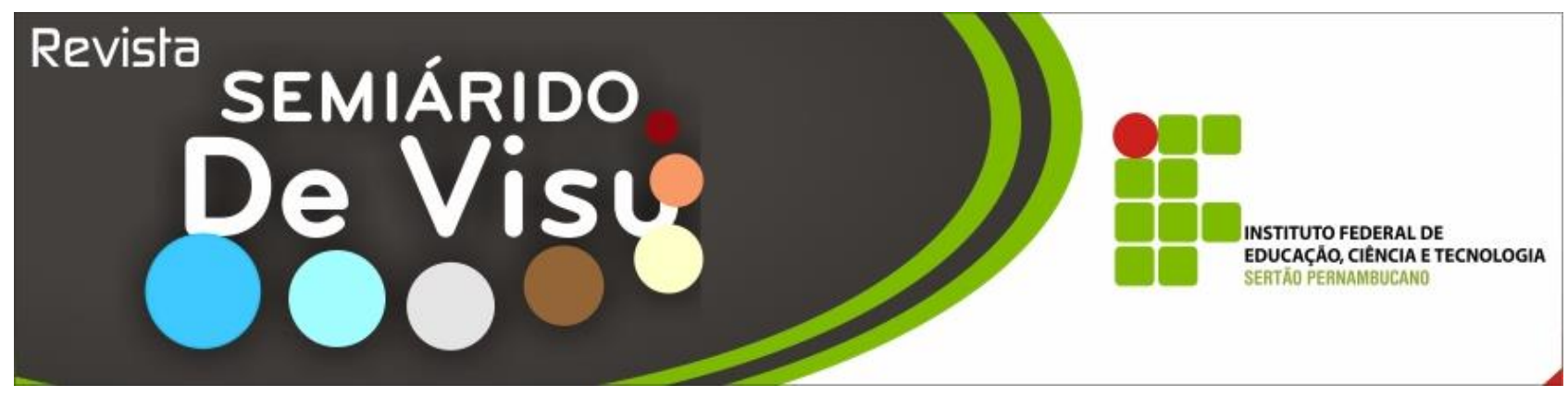

\title{
Determinação de um índice de conforto térmico para ovinos da raça Dorper
}

\author{
Aline Medeiros de Paula Mendes ${ }^{1}$, Marcilio de Azevedo², Paulo Roberto Cecon ${ }^{3}$, Guilherme Rocha \\ Moreira $^{4}$, Ângela Quintão Lana ${ }^{5}$ \\ ${ }^{1}$ Instituto Federal do Sertão Pernambucano - Campus Ouricuri. Estrada do Tamboril, s/n - Ouricuri - PE - Brasil. CEP: 56.200-000 / \\ Telefone: (87) 98125-2473 / E-mail: ${ }^{1}$ aline.mendes@ @ifsertao-pe.edu.br; \\ ${ }^{2}$ Universidade Federal Rural de Pernambuco - Departamento de Zootecnia, Campus Recife. Rua Dom Manoel de Medeiros, s/n, \\ Dois Irmão - Recife - PE - Brasil. CEP: 52.171-900 / Telefone: (81) 3320-6555/ E-mail: ${ }^{2}$ marcilio.azevedo@ufrpe.br; \\ ${ }^{3}$ Universidade Federal de Viçosa - Departamento de Estatística, Campus Viçosa. Avenida Peter Henry Rolfs, Campus Universitário \\ - Viçosa - MG - Brasil. CEP: 36.570 - 900 / Telefone: (31) 3899-1781/ E-mail: ${ }^{3}$ cecon@ ufv.br; \\ ${ }^{4}$ Universidade Federal Rural de Pernambuco - Departamento de Estatística e Informática, Campus Recife. Rua Dom Manoel de \\ Medeiros, s/n, Dois Irmão - Recife - PE - Brasil. CEP: 52.171-900 / Telefone: (81) 3320-6490/ E-mail: ${ }^{4}$ guirocham@ gmail.com; \\ ${ }^{5}$ Universidade Federal de Minas Gerais - Departamento de Zootecnia. Avenida Antônio Carlos, 6627 - Pampulha - Belo Horizonte - \\ MG - Brasil. CEP: 31270-901 / Telefone: (31) 3409-2202 / E-mail ${ }^{-5}$ angelaquintao@ gmail.com;
}

\begin{abstract}
Resumo: Foram observados vinte ovinos da raça Dorper, com idade média de 20 meses, machos, não castrados, no município de Arcoverde-PE, com o objetivo de determinar um índice de conforto térmico utilizando análise multivariada, e estimar seus níveis críticos. Os animais foram confinados em apriscos cobertos e avaliados à sombra, de manhã (6h30min.) e à tarde (12h30min.) e, os parâmetros fisiológicos aferidos foram temperatura retal (TR, $\left.{ }^{\circ} \mathrm{C}\right)$ e frequência respiratória (FR, mov $\mathrm{min}^{-1}$.). A equação obtida do índice de conforto térmico para ovinos Dorper foi: ICTD $=\mathrm{Ta} \times 0,46956+\mathrm{Tgn} \times 0,47194+\mathrm{vv} \times 0,19221$, em que Ta é a temperatura do ar $\left({ }^{\circ} \mathrm{C}\right)$, Tgn é a temperatura de globo negro $\left({ }^{\circ} \mathrm{C}\right)$ e vv é a velocidade do vento $\left(\mathrm{m} \mathrm{s}^{-1}\right)$. O ICTD foi comparado com o Índice de Temperatura e Umidade e o Índice de Conforto Térmico, apresentando correlações significativas com a FR ( $r=0,6910)$ e TR $(r=0,3360)$. Os níveis críticos estimados, para o ICTD foram 31,0 e 23,9, considerando a TR e FR como referência, respectivamente.
\end{abstract}

Palavras-chave: ambiência, bioclimatologia, estresse calórico, ovinos

\section{Determination of a thermal comfort index for Dorper sheep}

\begin{abstract}
Twenty Dorper sheeps were observed with a mean age of 20 months, males, intact, in the Arcoverde town, Pernambuco State, Brazil aiming to develop a thermal comfort index using multivariate analysis. The animals were maintained in covered installations in feedlot system and physiological reactions as rectal temperature $\left(\mathrm{RT}\right.$, $\left.{ }^{\circ} \mathrm{C}\right)$ and respiratory frequency (RF, mov $\mathrm{min}^{-1}$ ) were avaliated under shade in the morning (6h30min.) and afternoon (12h30min). The new equation of thermal comfort index is for Dorper sheep was ICTD $=$ Ta $\times 0.46956+\mathrm{Bgt} \times 0.47194$ $+\mathrm{vv} \times 0.19221$, where Ta is the air temperature $\left({ }^{\circ} \mathrm{C}\right) \mathrm{Bgt}$ is the black globe temperature $\left({ }^{\circ} \mathrm{C}\right)$ and vv is the wind speed $(\mathrm{m} / \mathrm{s})$. The index was compared with the Temperature and Humidity Index and Thermal Comfort Index, showing high correlations with RF $(r=0.6910)$ and $\mathrm{RT}(\mathrm{r}=0.3360)$. The estimated critical limits for the ICTD were 31.0 and 23.9 , based on the RT and RF respectively.
\end{abstract}

Key words: environment, bioclimatology, heat stress, sheep. 
(MENDES et al., 2017)

Introdução

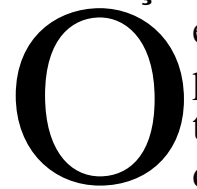

estresse pelo calor causado principalmente pelas elevadas temperaturas do ar reduz $\mathrm{o}$ desempenho e a eficiência reprodutiva de ovinos (Alhidary et al., 2012). Em virtude das limitações da temperatura do ar isoladamente como medida representativa do ambiente térmico, muitos esforços têm sido despendidos para combinar os efeitos de duas ou mais variáveis representando a influência das trocas de calor sensível e latente entre o animal e seu ambiente. Isso pode ser conseguido através de um índice de conforto térmico que representa o efeito produzido pelo processo de troca de calor, o qual pode alterar a resposta biológica que poderia estar associada com variações na temperatura do ar isoladamente (Hahn, 2009).

Índices de conforto térmico bem como seus níveis críticos são essenciais na elaboração de zoneamentos bioclimatológicos e a intensidade de participação de cada variável térmica ambiental na composição do índice é diferente para cada espécie animal, em razão das diferenças fisiológicas correspondentes (Medeiros, 2005).

De acordo com West (2003) índices de conforto térmico tem sido mais eficazes para avaliar o impacto do ambiente climático sobre os animais. O mais conhecido é o Índice de Temperatura e Umidade (ITU), desenvolvido por Thom (1958), para o conforto humano e tem sido amplamente empregado para avaliar o conforto térmico em bovinos (Azevedo et al., 2005) e ovinos (Maia et al., 2015; Tabarez-Rojas et al., 2009; Cezar et al., 2004). Santos et al. (2011), baseando-se nas variações de ITU, concluíram que o horário entre $12 \mathrm{~h} 00 \mathrm{~min}$ e $14 \mathrm{~h} 00 \mathrm{~min}$ é o mais estressante para ovinos a pasto. $O$ Índice de Temperatura de Globo e Umidade (ITGU) foi utilizado por Bezerra et al (2011) para avaliar o ambiente em estudos de adaptabilidade ao calor de diferentes grupos genéticos de ovinos no semiárido paraibano, onde as medidas registradas à tarde foram superiores aos registrados pela manhã.

Segundo Barbosa \& Silva (1995), índices para regiões específicas podem resultar em maior precisão na avaliação do ambiente para os animais. Esses autores desenvolveram um Índice de Conforto Térmico (ICT) para ovinos de raças exóticas criadas na região Sudeste do Brasil. Neves et al. (2009) concluíram ser o ICT adequado para avaliar o ambiente térmico para ovinos Santa Inês a pasto. O referido índice foi utilizado por Barbosa et al. (2001) no zoneamento bioclimatológico da ovinocultura no estado do Paraná.

Segundo Kubrusly (2001), ao desenvolver um índice é desejável que ele tenha a maior variância possível, ou seja, que contenha o máximo de informação fornecida pelo conjunto de variáveis selecionadas. Desse modo, no procedimento estatístico de análise multivariada, um método que cria combinações lineares com essa propriedade (máxima variância) é a análise de componentes principais.

É sabido que a raça ovina Dorper, originada na África do Sul, é direcionada para a produção de carne e requer regiões mais frias e úmidas (Mendes et al., 2014). Animais dessa raça têm sido introduzidos no nordeste do Brasil sem avaliação prévia sobre sua adaptabilidade ao calor para compor rebanhos puros ou para cruzamentos com raças nativas, no intuito de aumentar sua produtividade.

O objetivo deste trabalho foi estabelecer, através de análise multivariada, um índice de conforto térmico para os animais da raça ovina Dorper e estimar seus níveis críticos nas condições ambientais do semiárido nordestino.

\section{Material e Métodos}

$\mathrm{O}$ estudo foi conduzido de janeiro a março de 2011, na Fazenda Boa Vista, no município de Arcoverde, PE (08 $28^{\circ}$ '08" de latitude sul, $37^{\circ} 03^{\prime}$ ' 14 " de longitude oeste e 663 metros de altitude). De acordo com a classificação climática de Köppen adaptada para o Brasil, o clima de Arcoverde é o BSW' h', ou seja, muito quente, semiárido, com estação chuvosa de verão a outono, com temperaturas médias máximas mínimas de 
(MENDES et al., 2017)

29,02 e $18,3^{\circ} \mathrm{C}$, respectivamente e precipitação média anual de $575,7 \mathrm{~mm}$ (Encarnação, 1980).

As variáveis ambientais medidas foram a temperatura do ar (Ta) média, máxima e mínima, umidade relativa do ar (UR\%) média, máxima e mínima, temperatura de ponto de orvalho (Tpo), que foram transmitidos e armazenados por um sensor de temperatura e umidade sem Fio TFA Hygrotech 30.3125 e armazenados por um datalloger. A velocidade do vento $(\mathrm{Vv}, \mathrm{m} / \mathrm{s})$ foi medida através de um anemômetro digital portátil, e a temperatura de globo negro, por intermédio de um globotermômetro, colocado à $0,5 \mathrm{~m}$ acima do solo na a altura do dorso dos animais, ao sol e a sombra.

O Índice de Temperatura e Umidade (ITU) foi calculado utilizando-se a equação proposta por Thom (1958):

$$
I T U=T a+0,36 T p o+41,5
$$

em que: Ta é a temperatura do ar $\left({ }^{\circ} \mathrm{C}\right)$ e Tpo é a temperatura do ponto de orvalho $\left({ }^{\circ} \mathrm{C}\right)$.

$\mathrm{O}$ Índice de Conforto Térmico (ICT) para ovinos proposto por Barbosa \& Silva (1995) foi calculado pela equação:

$I C T=0,6678 \mathrm{Ta}+0,4969 P p\{t a\}+0,5444 \mathrm{Tgn}+0,1038$

Onde:Ta é a temperatura do ar $\left({ }^{\circ} \mathrm{C}\right), \operatorname{Pp}\{$ ta $\}$ é a pressão parcial de vapor $(\mathrm{kPa})$, Tgn é a temperatura do globo negro $\left({ }^{\circ} \mathrm{C}\right)$ e $\mathrm{Vv}$ é a velocidade dos ventos $(\mathrm{m} / \mathrm{s})$.

As variáveis ambientais consideradas para a determinação do Índice de Conforto Térmico para ovinos Dorper (ICTD) foram: Temperatura do ar (Ta), Temperatura de globo negro (Tgn) e Velocidade dos ventos (vv).

Foram observados 20 ovinos da raça Dorper, machos inteiros, com idade média de vinte meses. $\mathrm{O}$ estado sanitário dos animais estava dentro dos padrões de uma criação comercial, sendo o manejo de controle das doenças e parasitas realizado rotineiramente.

As observações de frequência respiratória (FR) e temperatura retal (TR), para a determinação do índice de conforto térmico, foram efetuadas à sombra, três vezes por semana, em dois horários, às $6 \mathrm{~h} 30$ e às $12 \mathrm{~h} 30 \mathrm{~min}$. A FR foi medida pela contagem dos movimentos dos flancos, com o auxílio de um cronômetro, durante 15 segundos, multiplicando-se o resultado por quatro.

A TR foi obtida por intermédio de um termômetro clínico digital, introduzido diretamente no reto do animal, até o acionamento do sinal sonoro.

Os dados foram submetidos à técnica de análise multivariada (análise de componentes principais) entre as variáveis dependentes (TR e FR) e as variáveis independentes (Ta, Tgn e vv).

Foram geradas equações, por meio de regressões múltiplas, para predição das variáveis fisiológicas (FR e TR) dos ovinos em função dos fatores ambientais (Ta, Tgn e vv).

Para as análises de regressão e correlação, foi utilizado o índice de conforto calculado com base nas variáveis ambientais obtidas nos horários de registro dos parâmetros fisiológicos, 6h30min e 12h30min.

Foram realizadas correlações entre as variáveis fisiológicas e os índices de conforto. Os níveis críticos dos índices de conforto foram obtidos por intermédio de análises de regressão simples, utilizando-se os dados fisiológicos e ambientais médios do período experimental. Foram escolhidos os modelos de regressão que melhor representaram as variações analisadas, baseando-se na resposta biológica, análise de variância da regressão e no valor do coeficiente de determinação $\left(R^{2}\right)$. Foi utilizado o pacote estatístico SAEG (2007) para todas as análises estatísticas.

Após o desenvolvimento do ICTD, este foi comparado com alguns dos índices de conforto térmicos mais utilizados como ITU e ICT por intermédio de análises de correlação entre esses índices e as respostas fisiológicas dos animais.

\section{Resultados e Discussão}


(MENDES et al., 2017)

Durante o período experimental os índices de conforto térmico variaram de 68,5 a 81,4 (ITU), 26,7 a 42,9 (ICT) e a temperatura do ar variou de 21,8 a $34,4^{\circ} \mathrm{C}$.
Através da análise multivariada de componentes principais foi determinado $\mathrm{o}$ Índice de Conforto Térmico para Dorper (Tabela 1):

Tabela 1. Análise multivariada de componentes principais (Explicação acumulada de 69\%).

\begin{tabular}{lccc}
\hline Variáveis ambientais & \multicolumn{3}{c}{ Componentes } \\
\cline { 2 - 4 } & 1 & 2 & 3 \\
Ta & 0.46956 & -0.21992 & -8.30493 \\
Tgn & 0.47194 & -0.19126 & 8.35542 \\
vv & 0.19221 & 1.00688 & -0.22675 \\
\hline
\end{tabular}

O primeiro componente, no caso, constitui o índice desejado, sendo:

$I C T D=T a \times 0,46956+T g n \times 0,47194+v v \times 0,19221$

em que:Ta $=$ temperatura do ar $\left({ }^{\circ} \mathrm{C}\right)$, Tgn $=$ temperatura do globo negro $\left({ }^{\circ} \mathrm{C}\right)$ e $\mathrm{vv}=$ velocidade dos ventos $(\mathrm{m} / \mathrm{s})$.
A eficiência dos componentes principais como índices de efeito ambiental, pode ser apreciada pelos coeficientes de correlação das variáveis ambientais com os componentes principais (Tabela 2).

Tabela 2. Correlações entre as variáveis ambientais e os componentes principais

\begin{tabular}{lccc}
\hline Variáveis & \multicolumn{3}{c}{ Componentes principais } \\
\cline { 2 - 4 } ambientais & 1 & 2 & 3 \\
Ta & 0,97794 & $-0,20015$ & $-0,05982$ \\
Tgn & 0,98289 & $-0,17407$ & 0,06018 \\
vv & 0,40030 & 0,91638 & $-0,00163$ \\
\hline
\end{tabular}

Pode-ser observar na Tabela 2, que o primeiro componente principal apresentou alta correlação com as variáveis climáticas e embora ele tenha mostrado ser um bom índice para conforto térmico dos ovinos Dorper, pode ser melhor calculado para determinada região ou macrorregião, porque as relações entre as variáveis ambientais são provavelmente diferentes de uma região para outra.

Na Tabela 3 observa-se que a correlação entre FR e TR foi positiva e significativa $(p<0,01)$, sugerindo que os animais utilizaram o aparelho respiratório para dissipação de calor e consequente manutenção do equilíbrio térmico.

Tabela 3. Coeficiente de correlação de Pearson entre as variáveis fisiológicas e os índices de conforto térmicos.

\begin{tabular}{cccccc}
\hline Variáveis & \multicolumn{5}{c}{ Correlações } \\
\cline { 2 - 6 } Fisiológicas & FR & TR & ITU & ICT & ICTD \\
FR & 1,0 & $0,2963^{* *}$ & $0,6947 * *$ & $0,6930^{* *}$ & $0,6910^{* * *}$ \\
TR & & 1,0 & $0,3416^{* *}$ & $0,3371^{* *}$ & $0,3360^{* *}$ \\
\hline
\end{tabular}

FR - Frequência Respiratória (mov./min.), TR - Temperatura Retal $\left({ }^{\circ} \mathrm{C}\right)$, ITU - Índice de

Temperatura e Umidade, ICT - Índice de Conforto Térmico e ICTD - Índice de Conforto Térmico para Dorper, $* *$ significativo a $1 \%$ de probabilidade $(\mathrm{P}<0,01)$. 
A TR e a FR se correlacionaram positiva e significativamente $(\mathrm{P}<0,01)$ com os índices de conforto e, de uma maneira geral, o coeficiente de correlação da FR foi maior que o da TR, sugerindo ser FR melhor indicador de estresse pelo calor que a temperatura retal, como pode ser observado na Tabela 3. Esses resultados foram também constatados, em ovinos das raças Ideal, Suffolk e Corriedale (Barbosa \& Silva, 1995), em ovinos Santa Inês de diferentes cores de pelame (Neves, 2008), em mestiços $1 / 2$ Dorper + $1 / 2$ SRD e $1 / 2$ Damara + 1/2SRD (Bezerra et al., 2011) e em mestiços $1 / 2$ Santa Inês $+1 / 2$ Dorper (Batista et al., 2014).

Animais homeotérmicos dispõem de vários mecanismos termorregulatórios cuja amplitude é altamente dependente da temperatura externa sendo o aumento na FR o primeiro sintoma visível do animal em estresse por calor (Silva, 2008). Severino et al. (2008) citaram que ovinos utilizam com mais intensidade o processo respiratório para manter a temperatura corporal. A correlação do ICDT com a TR $(0,3360)$ foi menor que aquela observada entre o ITU e a TR (0,3416). Barbosa \& Silva (1995) desenvolveram o ICT e a análise de correlação desse índice com a TR foi maior que a correlação entre a TR e o ITU. Por outro lado, a correlação da FR com o ICT obtida por esses autores, foi menor que com o ITU, enquanto que no presente trabalho os coeficientes de correlação foram similares. Assim sendo, e considerando ser a FR um melhor indicador de estresse calórico que a TR, pode-se inferir que o ICDT demonstrou ser um bom índice de conforto térmico para ovinos Dorper.

Também, apesar de não revelar superioridade sobre os outros índices como era esperado, o ICTD foi obtido nas condições ambientais da região semiárida, um fator relevante em relação aos outros índices.

Segundo Silva (2008), considera-se hipertérmico o animal que apresenta temperatura corporal maior que a média do lote mais um desvio padrão. Assim sendo, a temperatura retal a partir da qual os animais nesse estudo seriam considerados hipertérmicos foi de $39,7^{\circ} \mathrm{C}(39,5 \pm 0,2)$.

A análise de regressão (Figura 1) mostrou que o modelo linear foi o que melhor explicou as variações de TR dos animais em função do ICTD.

Pelo valor do coeficiente de determinação nota-se que os aumentos do ICDT podem explicar $11,29 \%$, das variações ocorridas na TR de ovinos. O restante das variações da TR pode ser explicado por fatores fisiológicos, nutricionais e comportamentais.

Figura 1.Temperatura retal (TR) dos animais em função do Índice de Conforto Térmico para Ovinos Dorper (ICTD).

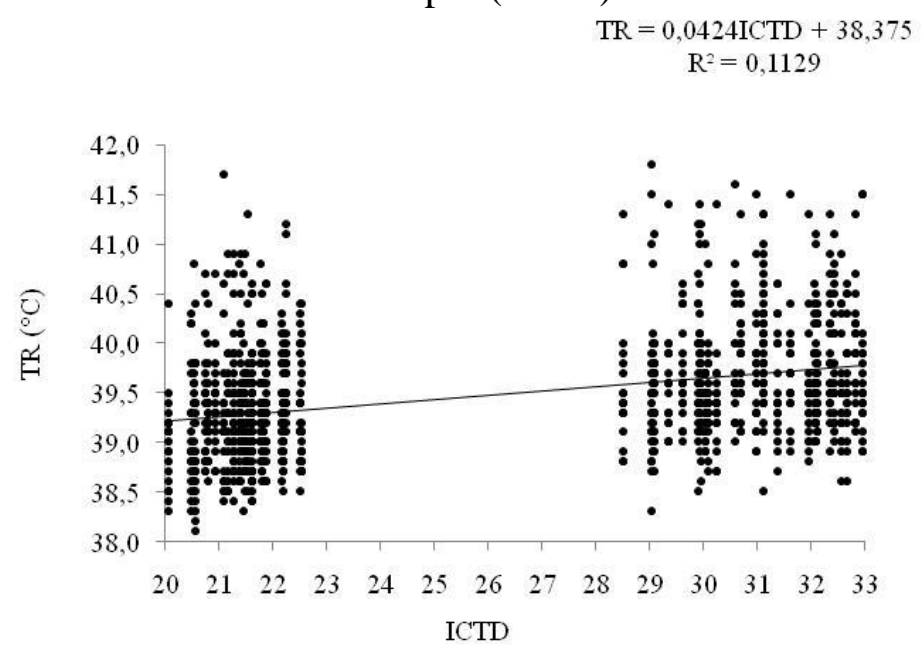

Fonte: Pesquisa direta. 
(MENDES et al., 2017)

Quando o ICTD aumentou de 26,2 para 31,0 a TR dos animais variou de $39,5^{\circ} \mathrm{C}$ para $39,7^{\circ} \mathrm{C}$. Estes animais tornaram-se hipertérmicos com ICTD acima de 31, podendo considerar este valor como crítico (Figura 1).

A amplitude de variação da FR observada durante o período experimental foi de 40 a 224 mov./min. Para a determinação do valor crítico dos índices de conforto em função da FR, estabeleceu-se a FR média destes animais como referência, conforme (Neves, 2008) sendo, portanto igual a 138,3 mov./min.

Figura 2 mostra a regressão da ICDT sobre a FR. O teste de ajuste de modelos mostrou que a equação que melhor representou as variações da FR dos animais em função deste índice, foi o modelo quadrático.

Figura 2. Frequência Respiratória (FR) dos animais em função do Índice de Conforto Térmico para ovinos Dorper (ICTD).

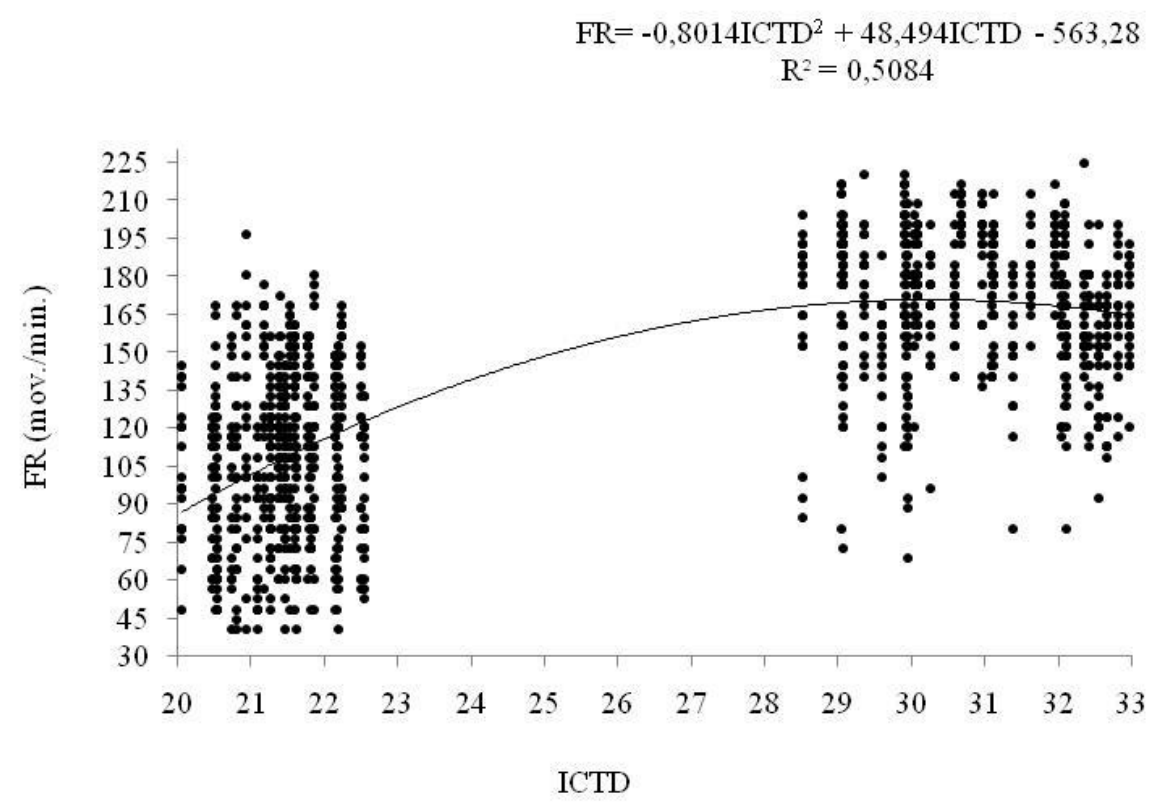

Fonte: Pesquisa direta.

Pelo valor do coeficiente de determinação nota-se que os aumentos do ICDT podem explicar 50,84\% das variações ocorridas na FR dos ovinos.

Com o ICTD de 26,2 os animais apresentaram FR de 157 mov./min (Figura 8). Com o aumento do ICTD para 31 a FR aumentou para 169,8 mov./min. O ICTD atingiu o valor máximode 30,3 quando a FR atingiu 170,3 mov./min. Os animais atingiram a média de FR $(138,3)$ com ICTD de 23,9, sendo este o valor crítico estimado para o Índice de Conforto Térmico para ovinos Dorper em sistema de confinamento, nas condições do sertão pernambucano.
Percebe-se que os valores críticos do ICTD quando se considera a FR como referência (Figura 2) são menores que aqueles observados quando a TR é considerada (Figura 1), significando que mecanismos homeostáticos, incluindo o aumento da FR, podem prevenir um apreciável aumento na TR antes que o índice de conforto atinja um ponto crítico. Este fato foi constatado também em vacas leiteiras por Lemerle \& Goddard (1986), Azevedo et al. (2005) e, em ovinos, por Neves et al. (2009), Bezerra et al. (2011) e Batista $t$ al (2014).

Segundo Silanikove (2000), a frequência respiratória pode quantificar a severidade do estresse pelo calor em 
ruminantes, em que uma FR de 40 - 60, 60 80 e 80 - 120 mov/min caracterizam, respectivamente, estresse baixo, médio-alto e alto, e acima de $200 \mathrm{mov} . / \mathrm{min}$. o estresse seria severo em ovinos. Baseando-se nessa classificação, pode-se inferir que nesse experimento foi observado nos animais estudados condições de estresse alto.

\section{Conclusões}

O Índice de Conforto Térmico para Ovinos Dorper demonstrou ser um bom índice em relação a outros mais comumente utilizados, tais como o Índice de Temperatura e Umidade e o Índice de Conforto Térmico.

Baseando-se na frequência respiratória e temperatura retal, o valor crítico do ICTD foi, respectivamente, de 23,9 e 31,0 valores esses que podem ser utilizados pelos criadores de ovinos da raça Dorper, no estado de Pernambuco, como referência para se definir o estresse pelo calor nos seus animais.

\section{Referências}

ALHIDARY, I. A.; SHINI, S.; AL JASSIM, R. A. M.; GAUGHAN, J. B. Physiological responses of Australian Merino wethers exposed to high heat load. Journal Animal Science, n. 90, p.212-220. 2012.

AZEVEDO, M.; PIRES, M.F.A.; SATURNINO, H.M.; LANA, A. M. Q.; SAMPAIO, I. B. M.; MONTEIRO, J. B. N.; MORATO, L. E. Estimativa de níveis críticos superiores do índice de temperatura $\mathrm{e}$ umidade para vacas leiteiras $1 / 2,3 / 4$ e $7 / 8$ Holandês-Zebu em lactação. Revista Brasileira de Zootecnia, v.34, n.6, p.20002008. 2005.

BATISTA, N. L.; SOUZA, B. B.; OLIVEIRA, G. J. C.; ROBERTO, V. B.; ARAÚJO, R. P.; RIBEIRO, T. L. A.; SILVA, R. A. Tolerância ao calor em ovinos de pelames claro e escuro submetidos ao estresse térmico. Journal Animal Behaviour Biometeorology, v.2, n.3, p.102-108, 2014.

BARBOSA, O.R.; SILVA, R.G. Índice de conforto térmico para ovinos. Boletim de Indústria Animal, v.52, n.1, p.29-35, 1995.

BARBOSA, O.R.; MACEDO, F.A.F.; GROES, R.V.; GUEDES, J. M. F. Zoneamento bioclimático da ovinocultura no estado do Paraná. Revista Brasileira de Zootecnia, v.30, n.2, p.454-460, 2001.

BEZERRA, W. M. A. X.; SOUZA, B. B.; SOUSA, W. H.; CUNHA, W. H. G. G.; BENÍCIO, T. M. A. Comportamento fisiológico de diferentes grupos genéticos de ovinos criados no semiárido paraibano. Revista Caatinga, v. 24, n. 1, p. 130-136, 2011.

CEZAR, M. F.; SOUZA, B. B.; SOUZA, W. H.; PIMENTA FILHO, E. C.; TAVARES, G. P.; MEDEIROS, G. X. Avaliação de parâmetros fisiológicos de ovinos Dorper, Santa Inês e seus mestiços perante condições climáticas do trópico semi-árido nordestino. Revista Ciência Agrotécnica, v.28, n.3, p.614- 620. 2004.

ENCARNAÇÃO, C.R.F. DA. Observações meteorológicas e tipos climáticos das unidades e campos experimentais da Empresa IPA. Recife: Empresa Pernambucana de Pesquisa Agropecuária, 117p. 1980.

HAHN, G.L., GAUGHAN, J.B., MADER, T.L., EIGENBERG, R.A. Thermal Indices and Their Applications for Livestock Environments. In: Livestock Energetics and Thermal Environmental Management. Ed J.A DeShazer. St. Joseph, USA, 2009. p. 113-130.

KUBRUSLY. L. S. Um procedimento para calcular índices a partir de uma base de dados multivariados.

Revista Pesquisa Operacional, v. 21, n. 1, p. 107-117. 2001.

LEMERLE, C.; GODDARD, M.E. Assemente of heat stress indairy cattle in 
Papua New Guinea. Topical Animal Health and Production, v.18, n.4, p.232-242, 1986.

MAIA, M. S.; SILVA, J. V. C.; MEDEIROS, I. M.; LIMA, C. A. C.; MOURA, C. E. B. Características seminais de carneiros das raças Dorper, Santa Inês e mestiços em condições de clima tropical. Revista Ciência Veterinária nos Trópicos, v. 18, n. 1 p. 2025. 2015.

MEDEIROS, C. M.; BAÊTA, F. C.; OLIVEIRA, R. F. M; TINÔCO, I.F. F.; ALBINO, L. F. T.; CECON, P. R. Índice térmico ambiental de produtividade para frangos de corte. Revista Brasileira de Engenharia Agrícola e Ambiental, v.9, n.4, p.660-665, 2005.

MENDES, A. M. P.; AZEVEDO, M.; LOPES, P. M. O.; MOURA, G. B. A. Zoneamento bioclimático para a raça ovina Dorper no Estado de Pernambuco. Revista Pesquisa Agropecuária Brasileira, v.49, n.12, p.986-993. 2014.

NEVES, M.L. M. W. Índices de conforto térmico para ovinos Santa Inês de diferentes cores de pelame em condições de pastejo. 77f. Dissertação (Mestrado em Zootecnia). Recife - PE: UFRPE, 2008.

NEVES, M. L. M. W.; AZEVEDO, M.; COSTA, L. A. B.; GUIM, A.; LEITE, A. M.; CHAGAS, J. C. Níveis críticos do Índice de Conforto Térmico para ovinos da raça Santa Inês criados a pasto no agreste do Estado de Pernambuco. Revista Acta Scientiarum Animal Sciences, v. 31, n. 2, p. 169-175, 2009.

SAEG. SAEG: Sistema para Análises Estatísticas, versão 9.1. Viçosa: UFV, 2007.

SANTOS, M. M.; AZEVEDO, M.; COSTA, L. A. B.; SILVA FILHO, F. P.; MODESTO,
E. C.; LANA, A. M. Q. Comportamento de ovinos da raça Santa Inês, de diferentes pelagens, em pastejo. Revista Acta Scientiarum Animal Sciences, v. 33, n. 3, p. 287-294, 2011.

SEVERINO, C. S. J.; FAÇANHA-MORAIS, D. A. E.; VASCONCELOS, A. M.; NERY, K. M.; MORAIS, J. H. G.; GUILHERMINO, M. M. Características termorreguladoras de caprinos, ovinos e bovinos em diferentes épocas do ano em região semiárida. Revista Científica Produção Animal. v. 10, n. 2, p. 127-137, 2008.

Silanikove, N. Effects of heat stress on the welfare of extensively managed domestic ruminants. Livestock Production Science, [S.1.], v.67, p.1-18, 2000.

SILVA, R. G. Biofísica Ambiental - Os animais e seu ambiente. Jaboticabal: Funep, 2008, 393p.

TABAREZ-ROJAS, A.; PORRASALMERAYA, A.; VAQUERA-HUERTA, H.; HERNÁNDEZ-IGNACIO, J.; VALENCIA, J.; ROJAS-MAYA, S.; HERNÁNDEZ-CERÓN, J. Desarrollo embrionário em ovejas Pelibuey y Suffolk em condiciones de estrés calórico. Revista Agrociencia, v. 43, n. 7, p. 671-679. 2009.

THOM, E.C. Cooling degree: Day air conditioning, heating, and ventilating. Transations of the Amer. Soc. Heating, Refrigerationg and Air-Conditioning Engrs. V.55, p.65-72, 1958.

WEST, J.W.; MULLINIX, B.G.; BERNARD, J.K. Effects of hot, humid weather on milk temperature, dry matter intake and milk yield of lactating dairy cows. Journal Dairy Science, v.86, n.6, p.232-242, 2003. 